\title{
Pathology and pathogenesis of liver disease in alpha-1-antitrypsin deficient individuals
}

\author{
Ø. Aagenaes \\ M.D. \\ K. ELGJo \\ M.D.
}

\author{
M. FAGERHOL \\ M.D. \\ E. Munthe \\ M.D.
}

SINCE the report of Laurell and Eriksson in 1963, in which they showed an increased frequency of emphysema in individuals with alpha-1-antitrypsin deficiency, this combination has been described by numerous authors. Eriksson (1965) mentioned that some of the emphysematic patients had cirrhosis of the liver. This was also found by others (Thuesen Pedersen, Weeke and Georg, 1969), but was initially assumed to be an unrelated coincidence. The report of Sharp et al. (1969) was the first to draw a definite relationship between liver disease and alpha-1-antitrypsin deficiency.

Sharp (1971) has continued his studies on liver disease in alpha-1-antitrypsin deficient children and several other reports have also confirmed the frequent finding of cholestasis and cirrhosis in these children (Johnson and Alper, 1970; Glasgow et al., 1971; Porter et al., 1972; Aagenaes et al., 1972; Talamo and Feingold, 1973).

The clinical picture in infancy is mainly one of cholestasis with jaundice, light stools and dark urine. Of our six patients in Oslo, three have been admitted with suspected biliary atresia and two have been admitted with umbilical bleeding. Laboratory findings are those of intrahepatic cholestasis, with increased transaminase, alkaline phosphatase, some increase in serum lipids, some steatorrhoea and an increase in serum bilirubin and bile acids.

Table 1 shows that in our last three patients where radioactive Rose Bengal tests have been performed, this test conformed well with an intrahepatic cholestasis, with a Rose Bengal excretion during the first 2 days between 10 and $20 \%$ of the injected amount. The duration of the cholestasis is most often a few months, and pruritis does not seem to be very severe.

After the cholestasis has subsided, the liver is still enlarged and firm and the enzymes remain somewhat increased. Thus far there have been few reports
Table 1. Alpha-1-antitrypsin deficiency with cirrhosis. Neonatal clinical picture

\begin{tabular}{ccc}
\hline Patient & $\begin{array}{c}\text { Clinical } \\
\text { cholestasis }\end{array}$ & $\begin{array}{c}\mathbf{J}^{131} \text { Rose Bengal } \\
\text { excretion }\end{array}$ \\
\hline 1 & + & \\
2 & + & \\
3 & + & $12 \%$ \\
4 & + & $15 \%$ \\
5 & + & $17 \%$ \\
6 & + & \\
\hline
\end{tabular}

Table 1 shows that all patients had a neonatal cholestasis of intra hepatic origin.

of an alpha-1-antitrypsin deficient child with neonatal cholestasis, where a later biopsy has shown a normalization of the liver. In all our patients there are signs of a slowly progressing cirrhosis. In a recent paper of Talamo and Feingold (1973), he reports improvement in the liver status after the first cholestatic period. This is an experience in all reported groups, but this does not indicate normalization. Our oldest patient is now 16 years old and in good condition, but with a definite cirrhosis and portal hypertension.

There is good evidence that infants with cholestasis have had the disease from foetal life.

In our six patients with cholestasis, average birth weight was $3000 \mathrm{~g}$, and this was also the average birth weight for the six patients reported by Porter et al. from King's College Hospital (1972). The alpha-1-deprived infants (Pi-type ZZ) with no signs of neonatal liver disease had a normal birth weight. As the births all were at term, the most reasonable explanation for the low birth weight is an already ongoing liver disease in the foetus.

There are no signs of other organs being affected, as for instance the brain. Laboratory examinations never showed signs of a generalized infection.

As no screening of alpha-1-antitrypsin infants has 


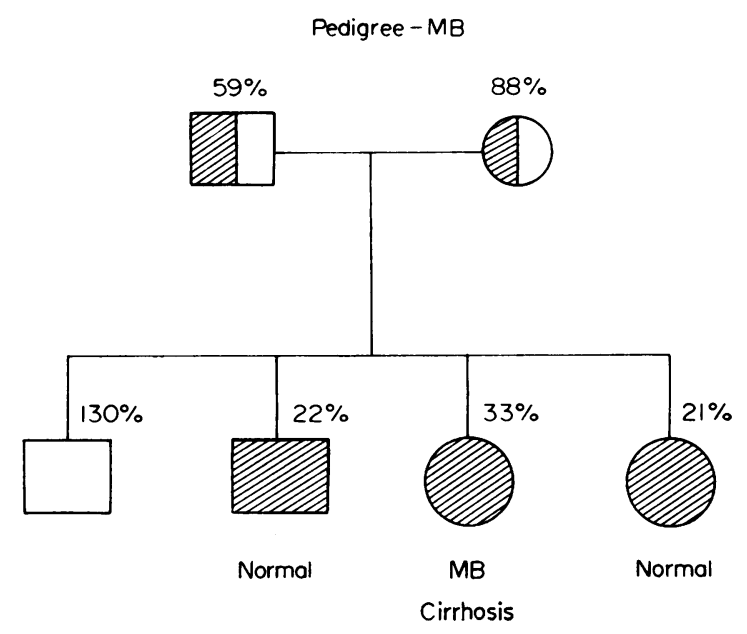

Fig. 1. Shows the alpha-1-antitrypsin concentration and the clinical picture in a family with three $\mathrm{ZZ}$ children. $\%=$ of normal alpha-1-antitrypsin concentration in serum. $\square$, male; $O$, female; $\square \mathrm{O}, \mathrm{Pi}$-type $\mathrm{MM}$; hatched/blank square and circle, Pi-type MZ; hatched square and circle, Pi-type ZZ.

been reported yet, we do not know exactly how many alpha-1-antitrypsin deficient children have clinical liver disease in infancy. In one of our families (Fig. 1) with three children with Pi-type ZZ, one child had clinical cholestasis and later signs of cirrhosis, and the other two children had no signs of liver disease.

Based on our own data and data from the literature, we suggest that the frequency of liver disease in alpha-1-antitrypsin deficient newborn is about $10-20 \%$.

The relative frequency of this type of liver disease in infants can be seen in Fig. 2. This shows that during a 3-year period at the Department of Pediatrics, Rikshospitalet, Oslo, we had twelve infants with biliary atresia, ten infants with intrahepatic cholestasis, of which four had alpha-1-antitrypsin deficiency, and fifteen patients with septicemia and signs of liver disease, most often cholestasis.

A few adults with emphysema have been found to have cirrhosis. In a recent work by Berg and Eriksson (1972) this has been found with such a high frequency at autopsy that it cannot be a coincidence, as had been thought earlier.

\begin{tabular}{lr}
\hline Atresia & 12 \\
Intrahepatic cholestasis (total) & \\
Familial giant cell transform & 2 \\
Alpha-1-antitrypsin deficiency & 4 \\
Other intrahepatic & 4 \\
Septicaemia with jaundice & 15 \\
(more or less cholestasis) & \\
\hline
\end{tabular}

Fig. 2. Shows the relative frequency of different neonatal cholestatic conditions in the Pediatric Department, Rikshospitalet. (3 years, 1968-1970.)

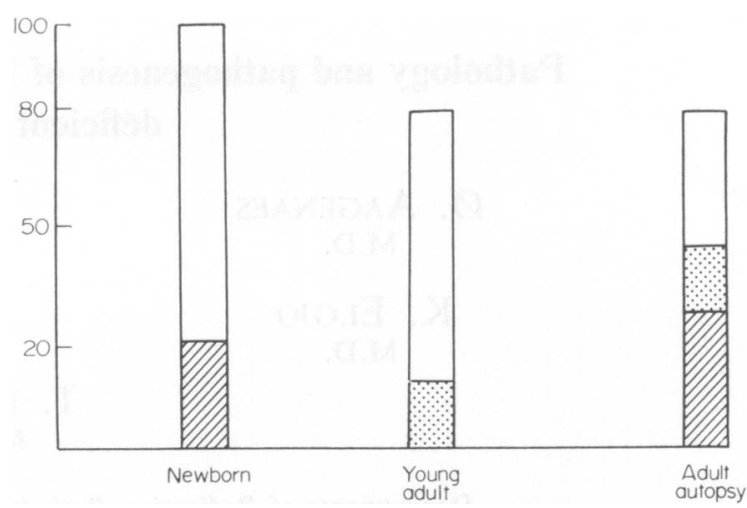

FIG. 3. Shows a rough estimation of the frequency of liver disease in $\mathrm{Pi}-\mathrm{ZZ}$ individuals at different ages. Hatched, cirrhosis; dotted, fibrosis.

Figure 3 shows the probable frequency of liver disease in alpha-1-antitrypsin deficient individuals at different ages: In infancy around $10-20 \%$ of the children have liver disease. These children will most often die before reaching adult age, and in young adult individuals with Pi-type ZZ, no frank cirrhosis has been reported so far, although slight fibrosis and a moderate increase in serum enzymes have been reported.

At autopsy, with an average age around 60 years, the frequency of cirrhosis in the liver is close to $40 \%$ according to Berg and Eriksson (1972).

After having prepared the slides and the text for this meeting, we have had some personal communications with Dr Tomas Sveger in Malmö, Sweden, where they have screened close to 80,000 newborn children and found more than forty $\mathbf{Z Z}$ individuals, of which three-four had clinical liver symptoms. This gives a somewhat lower frequency of neonatal liver disease in $\mathrm{ZZ}$ children than we had previously calculated. This is well explained by the fact that a ZZ-frequency found in Sweden of more than $1 / 2000$ is more than double the frequency found in Norway in adults (1/5000) (Fagerhol and Laurell, 1970). This genetic condition therefore seems also to be related to a relatively early death by causes other than liver disease.

Histological studies of livers in alpha-1-antitrypsin deficient individuals have given interesting results. First, they have shown that all individuals with Pitype $\mathrm{ZZ}$ have an intracellular material in their hepatocytes. In Fig. 4 we can see this material on this Hematoxylin-Eosin stained slide as yellow-brown intracellular pigment. These inclusions stain intensely red with PAS staining after diastase digestion, with the elimination of glycogen (Fig. 5).

Electron-microscopic studies of the inclusions (Fig. 6) show this to be an amorphous material that 


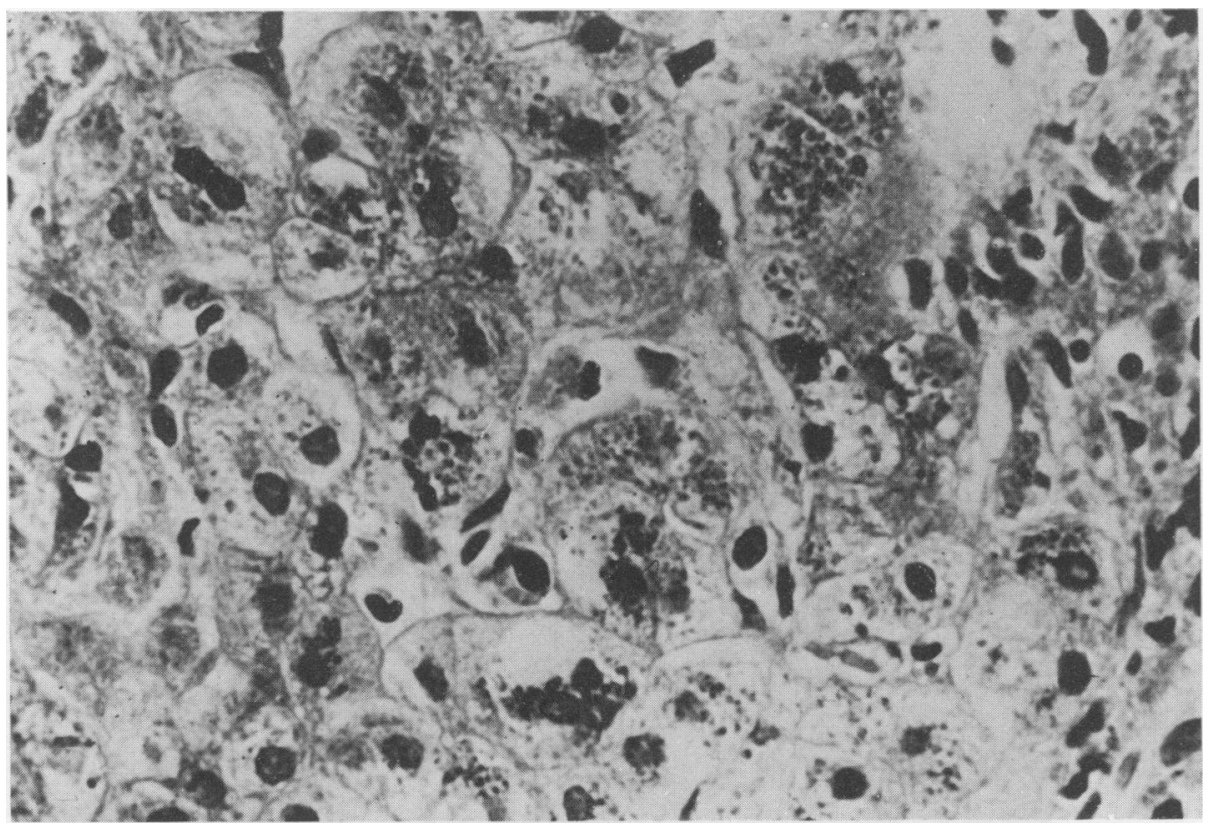

Fig. 4. (Female, age 1 year, liver disease, ZZ.) Many of the perilobular hepatocytes have a granular pigment in the cytoplasm, which stains yellow-brown with Haematoxylin-Eosin-Saffron.

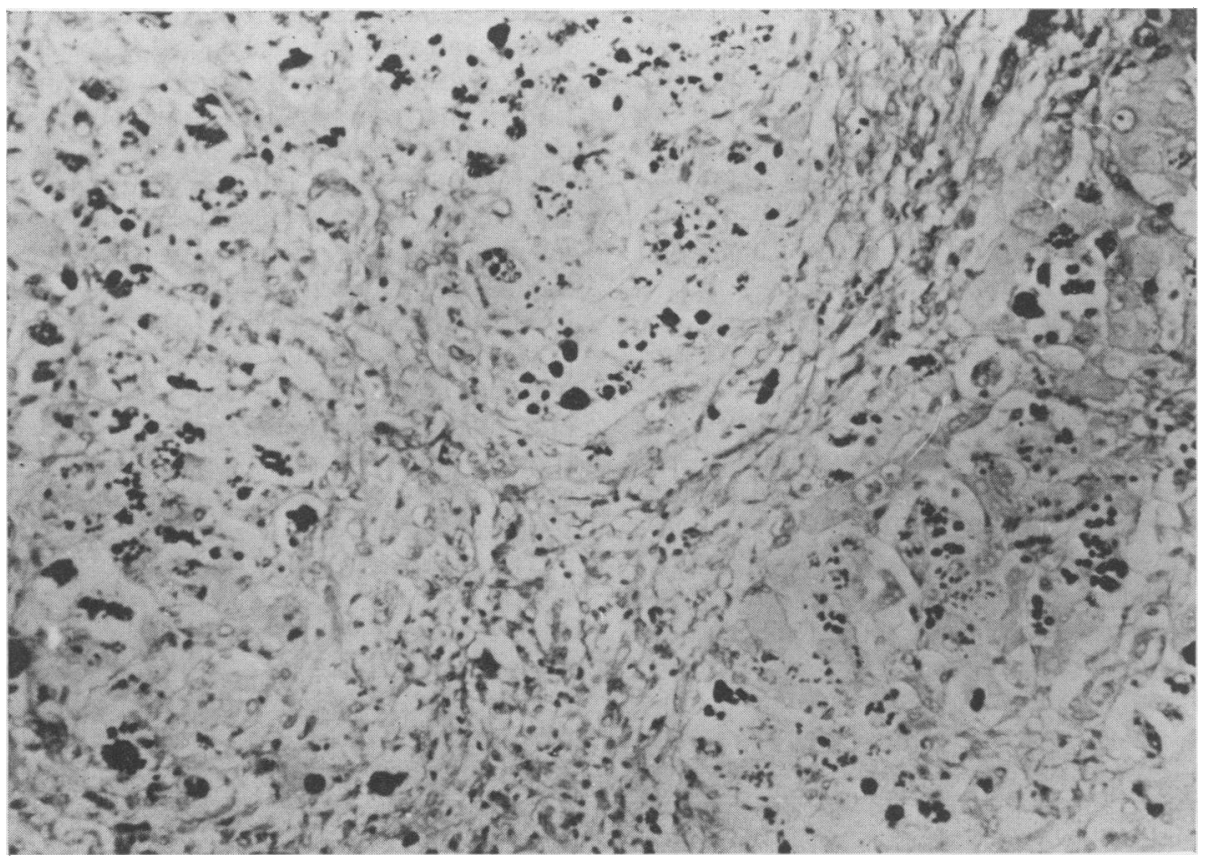

Fig. 5. (Male, age 45 years, cirrhosis, emphysema, ZZ.) Diastase resistant, MacManus positive globules in the hepatocytes. 


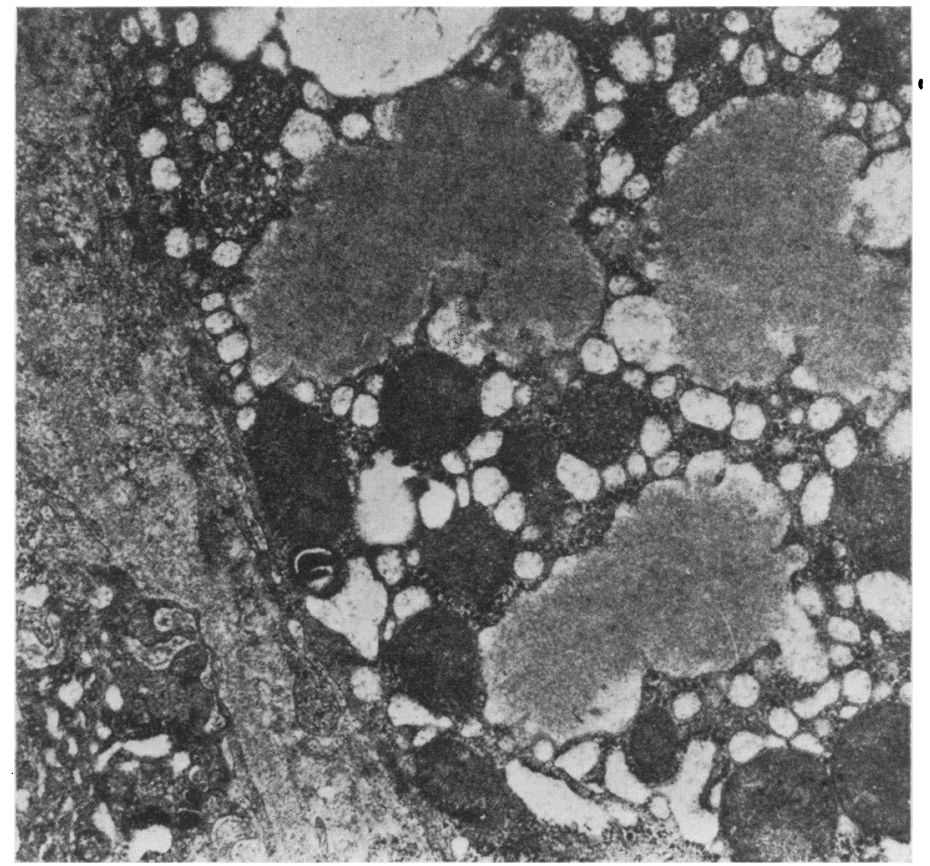

FIG. 6. Electronmicroscopy shows amorphous material in endoplasmatic reticulum $(\times 18,000)$.

is located intracellularly in small globules mainly located to the rough endoplasmatic reticulum.

When the biopsies were studied with fluorescein isotiacyanate labelled anti-alpha-1-antitrypsin, we can see a strong fluorescence intracellularly (Fig. 7).

Histochemical studies were also performed on some of the parents of our $\mathrm{ZZ}$ patients, with the $\mathrm{Pi}$ type $M Z$ (Fig. 8). As we can see here, they also showed some fluorescence specific for the alpha-1antitrypsin in the liver cells, but in fewer cells and with a lower titre than the $\mathrm{ZZ}$ individuals.

In a recent abstract from the last meeting of the European Association for the Study of the Liver, Feldman et al. (1973) report a study with horseradish peroxydase labelled anti-alpha-1-antitrypsin, where they can show by electron microscopy that the peroxidase is located to the amorphous substance in the endoplasmatic reticulum.

Table 2 shows a comparison between the alpha-1antitrypsin concentration in serum, the Pi-type, the highest dilution with which the fluorescence could still be seen, the percentage of liver cells showing fluorescence and the clinical picture. We can see that the dilution titre with which the fluorescence can still be seen and the percentage of liver cells showing fluorescence is closely related to the Pi-type and inversely related to the concentration of alpha-1antitrypsin in serum, but unrelated to the clinical state, liver cirrhosis, emphysema or normal status.
In infants with neonatal cholestasis, ordinary histology showed a cholestatic picture without any specific characteristics other than the earlier mentioned intracellular inclusions (Figs. 9 and 10). A pronounced piecemeal necrosis, hydropic degeneration of the liver cells and some derangement of the normal liver architecture are found, as well as bile duct proliferation, connective tissue proliferation and cellular infiltration in the portal region. There is no major giant cell transformation. The basal membrane between the portal area and the liver lobule is usually blurred.

Histological studies in adult alpha-1-antitrypsin deficient individuals will show intracellular globules, and histochemically we will see the alpha-1-antitrypsin, but elsewhere the liver is practically normal except for a very slight, unspecific portal fibrosis and slight degenerative changes (Fig. 11).

The next figure (Fig. 12) shows the pathological findings in an adult cirrhotic where frank portal cirrhosis occurs in $35-40 \%$ of the autopsies. In this diastase treated-PAS - stained section, we see the red intracellular material. Hepatoma is relatively frequent in these patients (Fig. 13).

As all $\mathrm{ZZ}$ individuals as well as $\mathrm{MZ}$ individuals have abnormal protein in their liver cells, and only some of the Pi-type $\mathrm{ZZ}$ individuals have liver disease, some additional factor must act to produce a liver disease in early infancy or in adult life. 


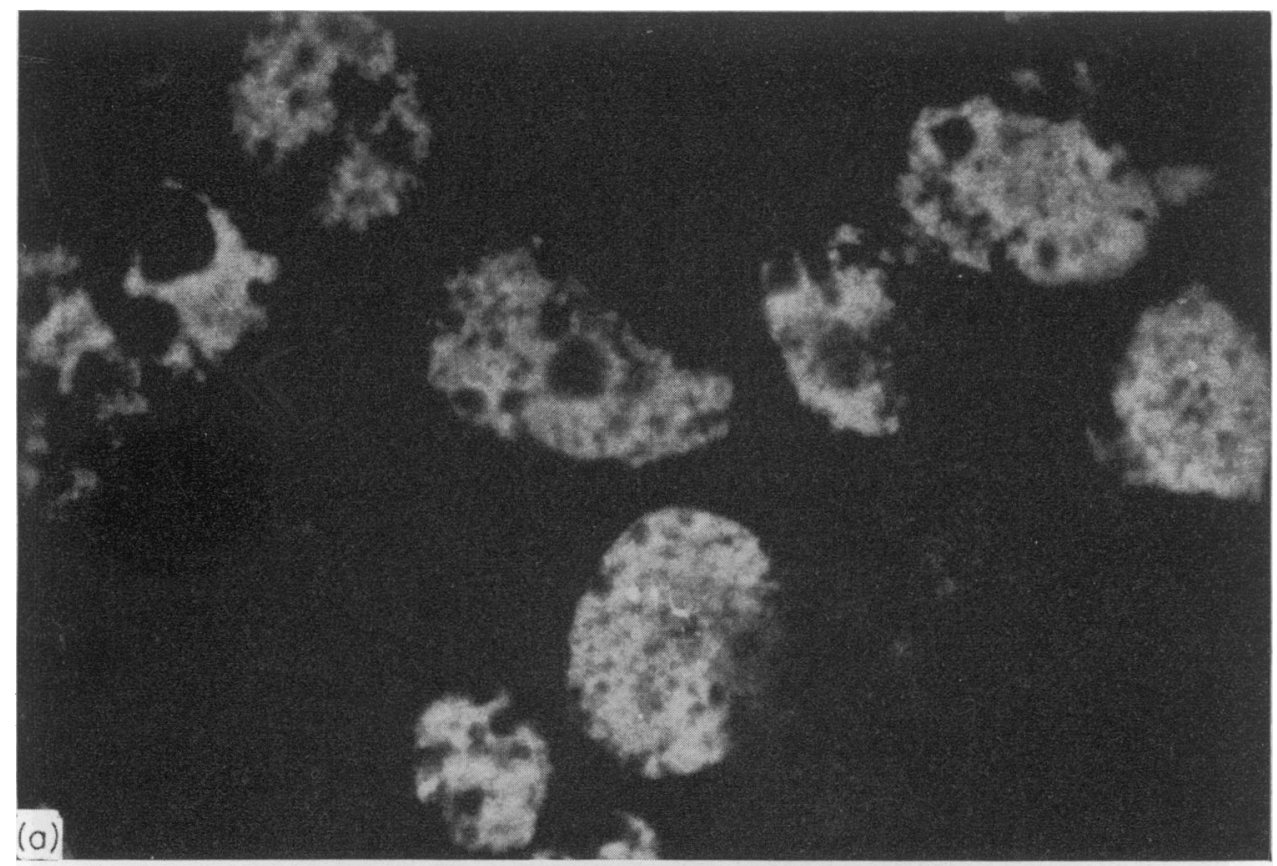

FIG. 7. (a) Liver section from ZZ homozygous patient, male 35 years old with no liver disease, stained for alpha-1-antitrypsin. Several hepatocytes are positively stained, but many hepatocytes are also negative. (b) Section from the same biopsy showing alpha-1-antitrypsin in larger deposits $(\times 1000)$. 

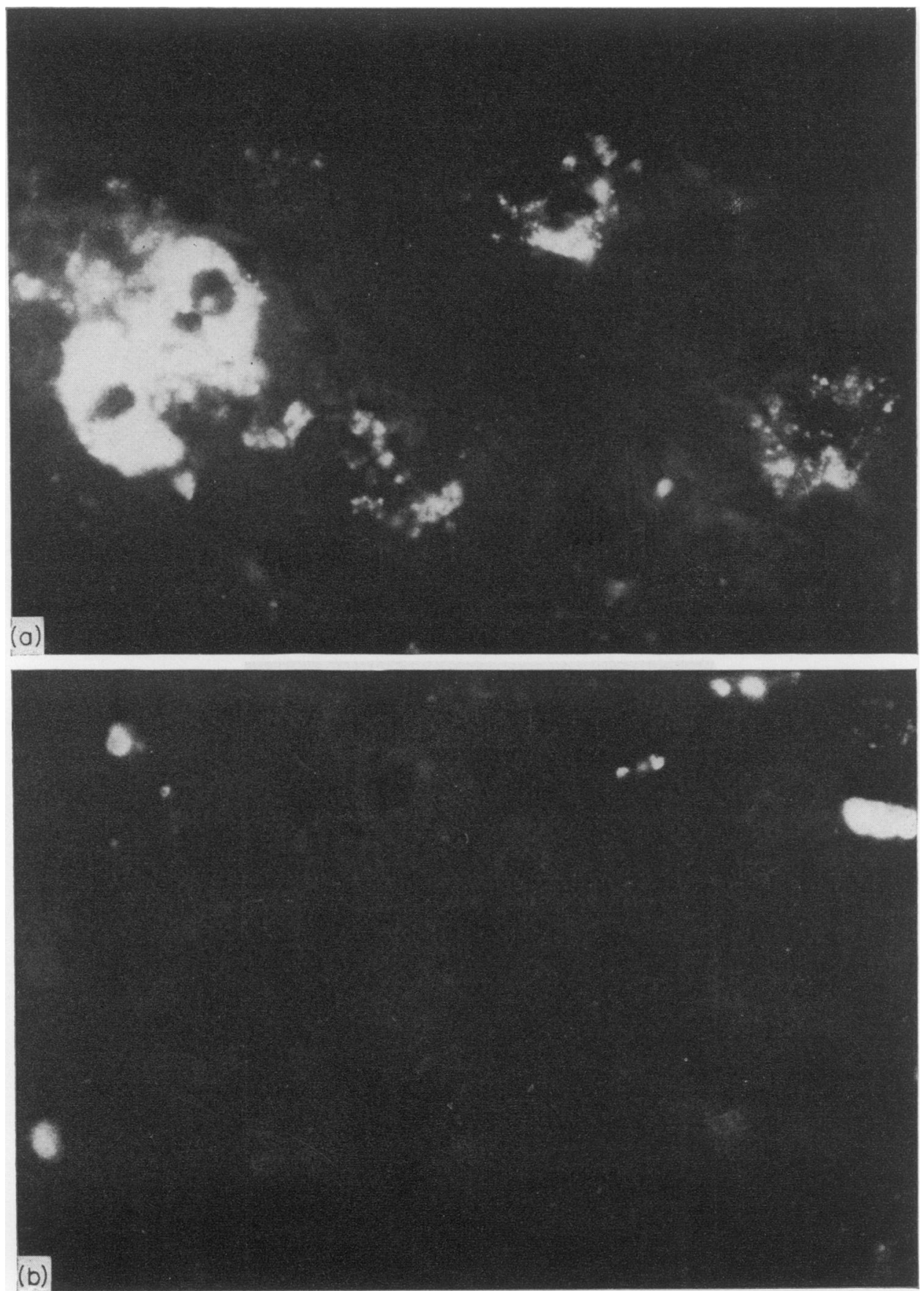

Fig. 8. (a) Liver section from MZ heterozygous patient, male 35 years old, showing a cluster of two hepatocytes staining positively with anti-alpha-1-antitrypsin.

(b) An area giving almost no positive staining in the same section. Such areas could also be found in tissues from ZZ homozygous patients $(\times 1000)$. 
TABLE 2. Comparison of alpha-1-antitrypsin content in serum and liver biopsies

\begin{tabular}{|c|c|c|c|c|c|}
\hline Case & Pi-type & $\begin{array}{l}\% \text { of normal } \\
\text { serum conc. }\end{array}$ & $\begin{array}{l}\text { Highest dilution } \\
\text { of conjugate } \\
\text { giving positive } \\
\text { tissue reaction }\end{array}$ & $\begin{array}{l}\text { Approx. } \% \\
\text { pos. } \\
\text { hepatocytes }\end{array}$ & $\begin{array}{c}\text { Clinical } \\
\text { status }\end{array}$ \\
\hline A.N.K. & $\mathbf{Z Z}$ & 27 & $1: 256$ & $60-70$ & cirrhosis \\
\hline J.I.J. & $\mathbf{Z Z}$ & 18 & $1: 512$ & $40-60$ & cirrhosis \\
\hline C.E. & $\overline{\mathbf{Z Z}}$ & 41 & $1: 512$ & $40-60$ & cirrhosis \\
\hline G.K. & $\overline{\mathbf{Z Z}}$ & 20 & $1: 512$ & $20-40$ & emphysema \\
\hline I.M.O. & $\mathbf{Z Z}$ & 45 & $1: 512$ & $20-40$ & normal \\
\hline A.B.B. & $\mathrm{ZZ}$ & 21 & $1: 256$ & $50-70$ & normal \\
\hline G.B. & $\overline{\mathrm{ZZ}}$ & 22 & $1: 256$ & $50-70$ & normal \\
\hline V.E. & $\mathbf{M Z}$ & 75 & $1: 128$ & $1-5$ & normal \\
\hline L.J. & $\mathbf{M Z}$ & 68 & $1: 256$ & $1-5$ & normal \\
\hline E.J. & $\mathbf{M Z}$ & 50 & $?$ & 0 & normal \\
\hline
\end{tabular}

Table 2 shows that there is a reverse relationship between the alpha-1-antitrypsin concentration in serum and the frequency of positive hepatocytes as well as the dilution titer that still gives positive fluorescence in the liver cells.

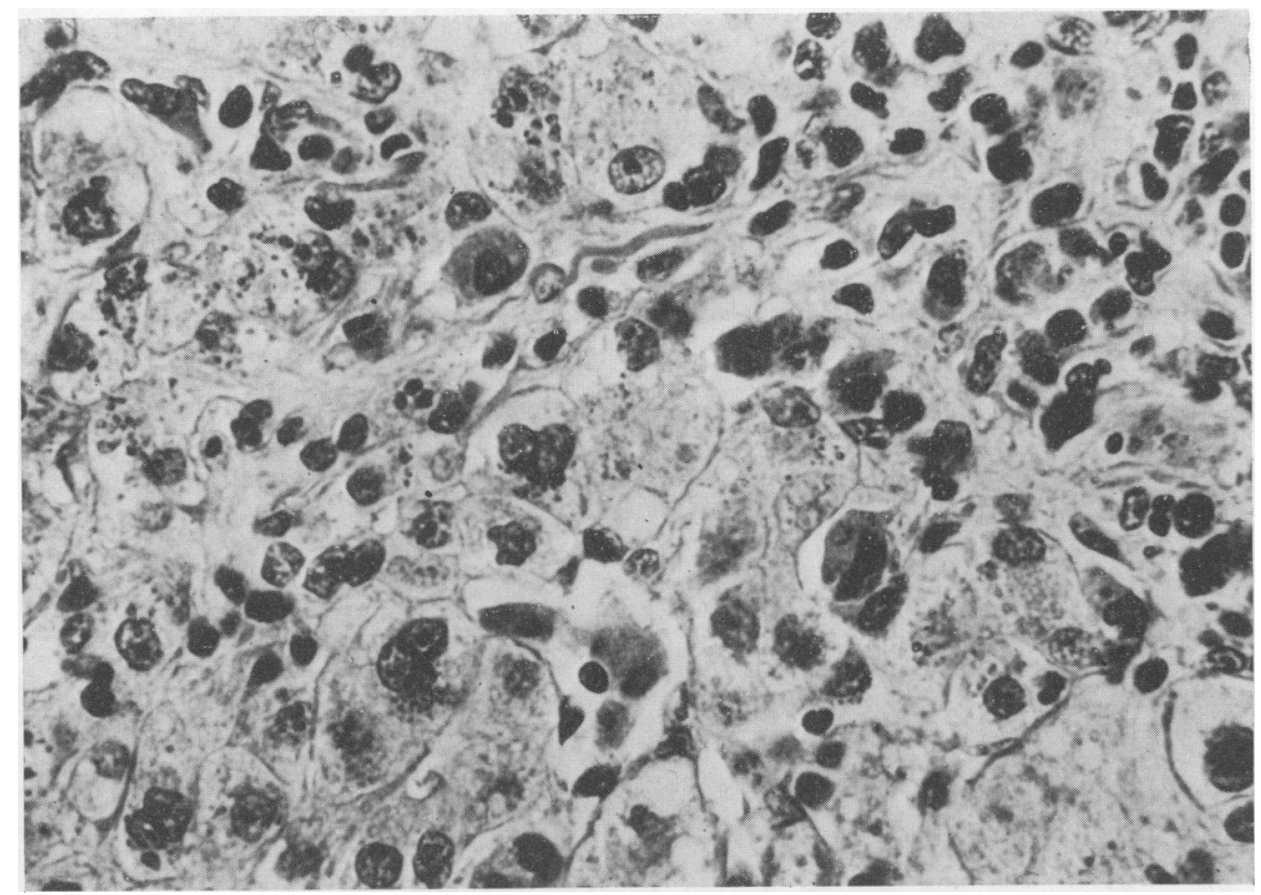

FIG. 9. (Male, 3 months old, $\mathrm{ZZ}$, liver disease.) This figure shows a blurred basal plate, increased connective tissue in the sinusoids and different stages of necrosis of the hepatocytes-also with some eosinophilic bodies. (Haematoxylin-Eosin-Saffron.)

Figure 14 shows possible additional factors. An infectious factor, as for example serum hepatitis virus, has been thought to be a possible additional factor in a study from King's College Hospital (Porter et al., 1972) because of a relative frequent finding of Australia antigen in the infants and their parents. All our patients have been Australia antigen negative. The parents have not been examined.
Personal communications from Sharp in Minneapolis indicate that he has found no Australia antigen positive infants in his groups. We think there are several indications that serum hepatitis virus is not the actual additional factor.

(1) So far most of the reported infants who have received Australia antigen positivity from their $\mathrm{Au}-$ antigen positive, but not diseased mothers, seem to 


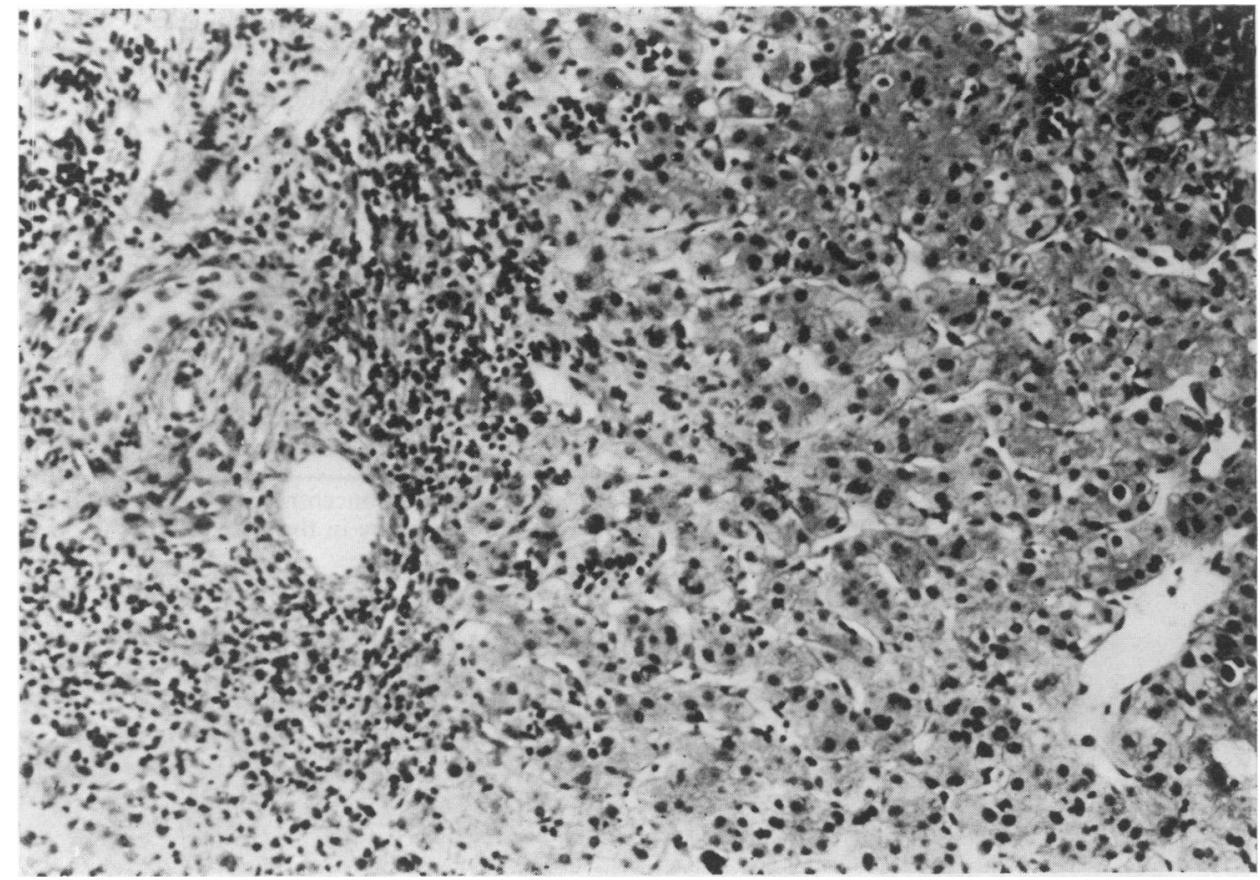

Fig. 10. In this section of the same liver as Fig. 9, the architecture of the liver lobule is relatively normal, but we see a pronounced cellular infiltration and increase in connective tissue. A central vein is seen in the right corner, and a portal area in the left $1 / 3$.

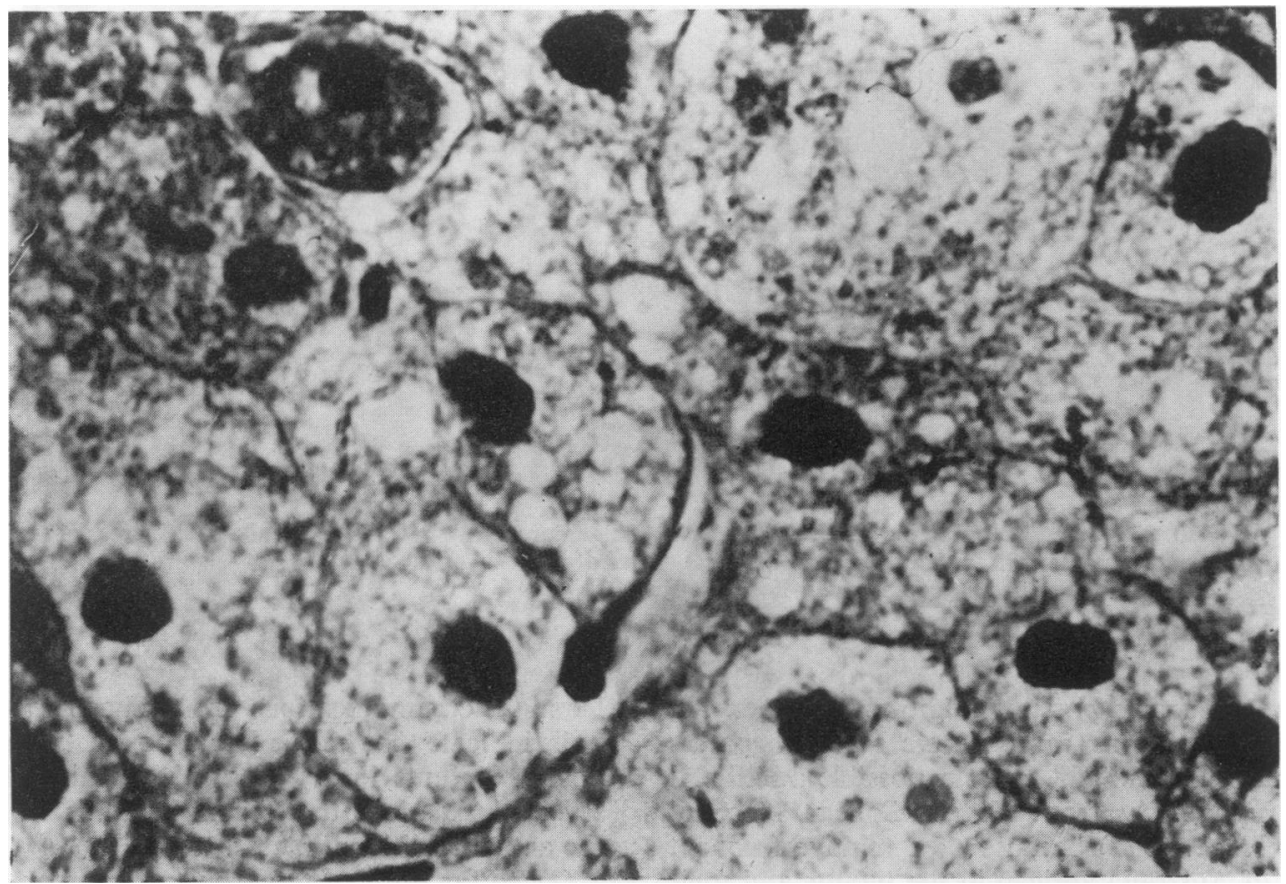

FIG. 11. (Female, 35 years old, ZZ, no liver disease.) This high power (oil immersion) microphotograph shows enlarged and degenerated hepatocytes and one acidophil body in a sinusoid. Some hepatocytes contain small granules with the same staining properties as those found in the biopsies from children (Fig. 4). 


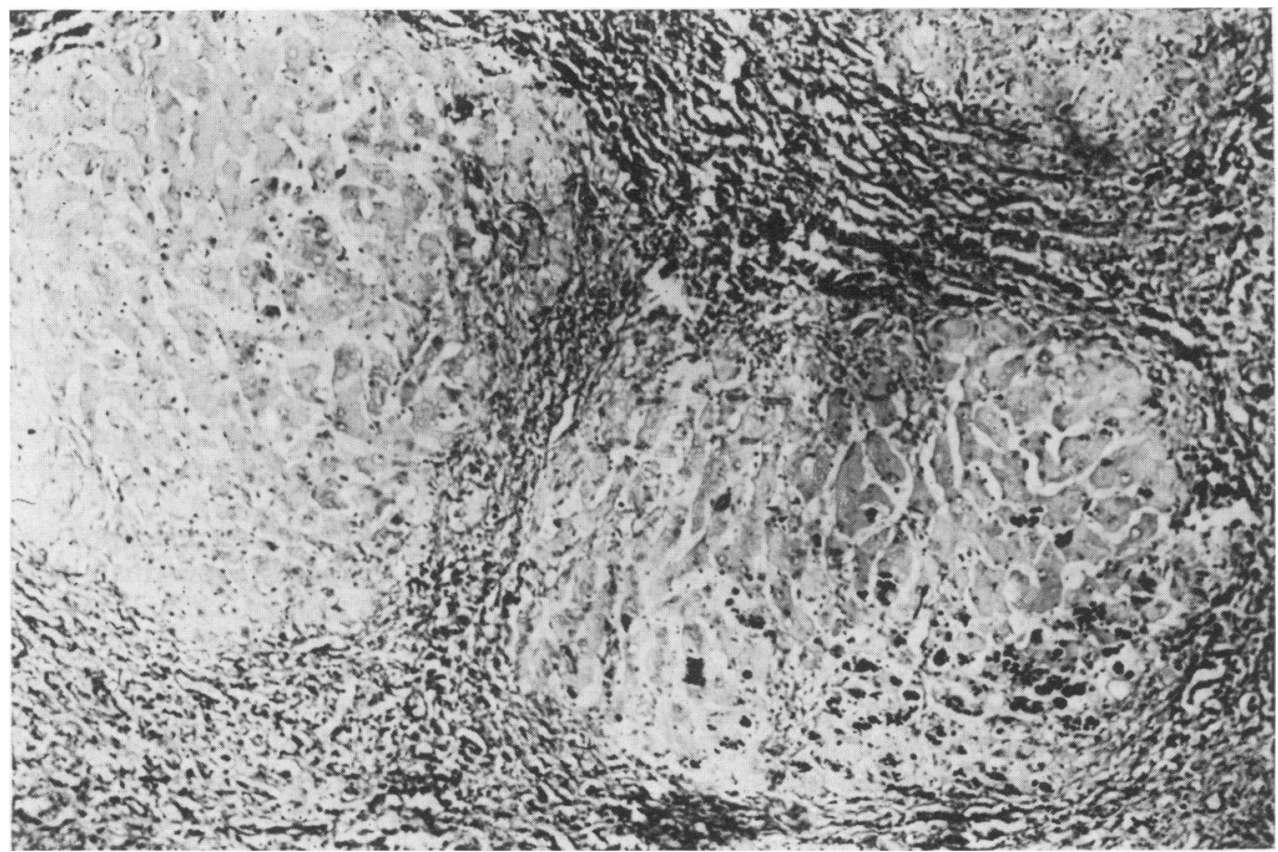

FIG. 12. (Male, 45 years old, cirrhosis, emphysema, ZZ.) Red, intracellular globuli in diastase treated, MacManus-stained section.

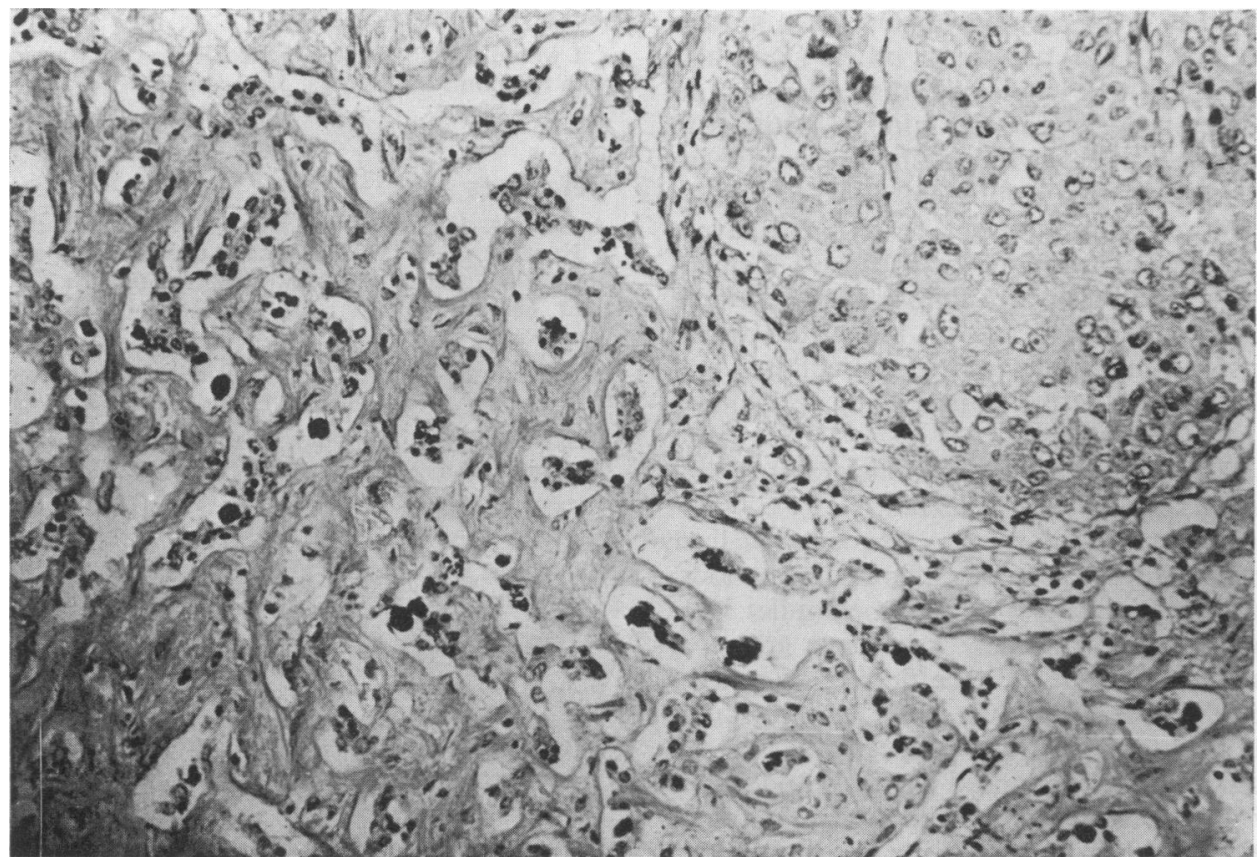

FIG. 13. (Male, 49 years old, cirrhosis and cancer hepatis, ZZ.) Hepatocellular cancer without globuli and compressed liver cells with globuli (MacManus after diastase). 


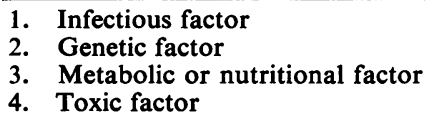

FIG. 14. Possible additional factors in pathogenesis of liver disease in Pi-type $\mathrm{ZZ}$ individuals.

have got this after birth and not through the placenta. From our findings of the low birth weight in the alpha-1-antitrypsin deficient liver patients, there seems to be little doubt that they have had an intrauterine liver disease.

(2) The clinical picture of a severe cholestasis soon after birth has thus far not been found in any infant who has contracted Australia antigen from the mother. In these infants the clinical symptoms are very vague - or completely lacking - and start 1-2 months after birth.

(3) If the Australia antigen or serum hepatitis virus were an additional factor, we should also expect an increased frequency of serum hepatitis in adult $\mathbf{Z Z}$ patients, and this has not been reported so far.

There is no apparent reason for suspecting other infectious agents during intrauterine life. All the mothers have been well during pregnancy without periods of fever and the finding of infectious agents such as rubella virus, toxoplasma gondii, cytomegalovirus and others, has never been reported.

Genetic factors other than the alpha-1-antitrypsin deficiency have to be considered. Our own studies (Aagenaes et al., 1972) as well as others (Berg and Eriksson, 1972) have indicated that there is no genetic difference as to the alpha-1-antitrypsin deficiency in the patients with childhood liver disease and in the patients with emphysema. The possibility that the children with the liver disease could have a double gene defect, with the liver disease then inherited by a linked genetic abnormality, must be considered. Based on our present knowledge where liver disease is found both in newborn children and older adult patients with Pi-type ZZ, this possibility does not seem very likely.

The third possibility, of a metabolic or nutritional factor, must therefore be considered.

Figure 3 shows us the relative frequency of liver disease in ZZ-individuals in the different age periods.

As liver function studies or enzyme studies have not been performed routinely in a large group of individuals with Pi-type $\mathrm{ZZ}$, the finding of a slight increase in the liver enzymes in some $\mathrm{ZZ}$ individuals might just be intercurrent and unspecific.

The finding of a pronounced cirrhosis and also a relatively high frequency of hepatomas in older adult patients with a long duration of emphysema, must in one way or another be related to the alpha-1antitrypsin deficiency. The emphysema per se can give slight central fibrosis but no real cirrhosis. We therefore feel that we have to look for a commonc. factor in the foetal period and in the period of severe emphysema. One factor might be a lower than normat? $P_{2}$, but many other factors might also be responsible In some way or another, we might suggest that eithero the presence of the abnormal alpha-1-antitrypsin in the liver cell or the low value of alpha-1-antitrypsin $\mathbb{R}$ in the serum with an additional factor in foetal lifees or in a severe emphysematic condition will cause ametabolic or nutritional damage to the liver celf followed by the development of cirrhosis.

As to the last point on the figure, we have nog evidence of any toxic factor that might be the additional factor.

In conclusion then, the accumulation of alpha-1 antitrypsin or a slightly abnormal alpha-1-antier trypsin in the liver cells of $\mathrm{ZZ}$ individuals is there $-\stackrel{\infty}{\perp}$ fore most probably due to the synthesis of a struc turally different protein because of a mutation af the locus for the alpha-1-antitrypsin structural genes음 The liver cells are unable to secrete this abnormat protein. This is in accordance with a recent repore (Gordon et al., 1972) that even subjects homozygoted for another variant, Pi S, show a similar accumula $\overrightarrow{0}$ tion. With an additional metabolic or nutritiogat factor, the liver becomes damaged and the cirrhosis develops.

\section{Acknowledgment}

I would like to thank Dr Sten Eriksson, Malmo, Sweden? who has contributed Figs. 5, 13 and 14.

\section{References}

Aagenaes, Ø., Matlary, A., Elgjo, K., Munthe, E. FAGERHOL, M. (1972) Neonatal cholestasis in alpha-1-antị. trypsin deficient children. Acta Paediatrica Scandinavica 61, 632.

BERG, N.O. \& ERIKSSON, S. (1972) Liver disease in adulti with alpha-1-antitrypsin deficiency. The New Englang Journal of Medicine, 287, 1264.

ERIKSSON, S. (1965) Studies in alpha-1-antitrypsin deficiency Acta Medica Scandinavica, 177, Suppl. 175.

Eriksson, S. \& Hagerstrand, I. (1973) Acta Medic Scandinavica (in press).

Fagerhol, M.R. \& Laurell, C.-B. (1970) The Pi system or inherited variants of serum alpha-1-antitrypsin. In Progress in Medical Genetics, Vol. VII, pp. 96-111. Grunछ \& Stratton, New York.

Feldmann, G., Bignon, J., Chahiman, P., Guesnon, J. \& GiLeT, M. (1973) Identification of alpha-1-antitrypsin if the liver cells of patients with alpha-1-antitrypsin de ficiency: an ultrastructural and immuno-cytochemicat study. Abstract, European Association Study Liver, Vitelled

Glasgow, J.F.T., Hercz, A., Levison, H., LyNCH, M.J. \& SASS KORTSAK, A. (1971) Alpha-1-antitrypsin deficienc\$ with both cirrhosis and chronic obstructive lung disease i two sibs. Pediatric Research, 5, 427. 
Gordon, H.W., Dixon, J., Rogers, J.C., Mittman, C. \& Lieberman, J. (1972) Alpha-1-antitrypsin accumulation in livers of emphysematous patients with alpha-1-antitrypsin deficiency. Human Pathology, 3, 361.

Johnson, A.M. \& AlPER, C.E. (1970) Deficiency of alpha-1antitrypsin in childhood liver disease. Pediat rics, 46, 921.

LAUrell, C.B. \& ERIKSSON, S. (1963) The electrophoretic alpha-1-globulin pattern of serum in alpha-1-antitrypsin deficiency. Scandinavian Journal of Clinical and Laboratory Investigation, 15, 132.

Porter, C.A., Mowat, S.P., Cook, P.J.L., Haynes, D.W.G., Shilkin, K.B. \& Williams, R. (1972) Alpha-1-antitrypsin deficiency and neonatal hepatitis. British Medical Journal, 3, 435 .
Sharp, H., Bridges, R. A., Krivit,W. \& Freier, E.F. (1969) Cirrhosis associated with alpha-1-antitrypsin deficiency. The Journal of Laboratory and Clinical Medicine, 73, 934.

Sharp, H. (1971) Alpha-1-antitrypsin deficiency. Hospital Practice, 5, 83.

TAlamo, R.C. \& Feingold, M. (1973) Infantile cirrhosis with hereditary alpha-1-antitrypsin deficiency. American Journal of Diseases in Childhood, 125, 845.

Thuesen Pedersen, J., Weeke, B. \& Georg, J. (1969) Lung disease and alpha-1-antitrypsin deficiency. Danish Medical Bulletin, 16, 283.

\section{Discussion}

In answer to questions by Professor Emery, Professor Aageneas stated that it had not been possible to examine the placentas of any of these infants for histological abnormality.

Professor Alagille from Paris commented that he had studied twenty-five patients with liver disease and alpha-1-antitrypsin deficiency. Three of these were heterozygous $\mathrm{MZ}$, one of whom had severe portal hypertension at the age of 3 years. One patient has a Pi SZ phenotype, one patient with $\mathrm{Pi} \mathrm{ZZ}$ phenotype had clinical and histological features of intrahepatic bile duct hypoplasia. In none of the twentyfive patients was hepatitis B antigen found. Professor Aagenaes had also had one patient with Pi-MZ pheno- type with liver disease having a Banti syndrome with portal hypertension but because the $M Z$ phenotype is so relatively common, he did not consider that it was necessarily related to the $\mathrm{MZ}$ state.

Dr Tavill of London commented that to his knowledge this was the first example of a disease where the failure of secretion of a protein could be involved and asked whether the alpha-1-antitrypsin within the liver cells was, in fact, normal. Professor Aagenaes commented that although there were some studies which suggested there was some abnormal carbohydrate in the glyco-protein, this had not been confirmed. 